\title{
Aumento do tempo de permanência dos turistas no Brasil: Novos turistas ou novos comportamentos?
}

\author{
Increase of tourists' length of stay in Brazil: \\ New tourists or new behaviors?
}

\author{
Crecimiento del tiempo de estada de los turistas en Brasil: \\ ¿Nuevos turistas o nuevos comportamientos?
}

\author{
Glauber Eduardo de Oliveira Santos ${ }^{1}$ \\ Vicente Ramos \\ Javier Rey-Maquieira ${ }^{2} *$
}

Resumo: Em todo o mundo a duração média das viagens tem diminuído. No entanto, curiosamente, essa tendência não tem se revelado no turismo receptivo internacional do Brasil. Duas hipóteses podem explicar a incomum realidade brasileira: mudanças no comportamento dos turistas que visitam o país, e alterações na composição do fluxo turístico receptivo internacional brasileiro. Uma eventual confirmação da primeira hipótese mostraria que a evolução do comportamento dos turistas nos últimos anos foi efetivamente diferente no Brasil e nos demais países, evidenciando a ocorrência de melhorias na qualidade da experiência turística ofertada pelo Brasil. Este estudo objetiva testar essa hipótese através do uso de modelos de duração baseados em mais de 211 mil entrevistas. A modelagem do comportamento individual dos turistas torna possível controlar o efeito de variáveis intervenientes, permitindo uma avaliação detalhada do efeito temporal sobre a duração esperada das estadas dos turistas. Treze modelos de duração são testados. O modelo com melhor ajuste aos dados tem taxa de risco com distribuição Weibull e heterogeneidade individual com distribuição Gama. Os resultados apontam que a tendência crescente da permanência média é parcialmente explicada por uma mudança no comportamento dos turistas.

Palavras-chave: Permanência; Duração; Estada; Modelos de duração; Turismo no Brasil.

Abstract: The average duration of tourism trips has decreased all over the world. However, curiously, this trend has not been observed in the Brazilian inbound tourism. There are two hypotheses for explaining

\footnotetext{
${ }^{1}$ Bacharel e Mestre em Turismo pela Escola de Comunicações e Artes da Universidade de São Paulo. Doutorando em Economia do Turismo e do Meio Ambiente pela Universidade das Ilhas Baleares (Espanha). Professor do Instituto Federal de Educação, Ciência e Tecnologia de São Paulo (IFSP). E-mail: glaubereduardo@gmail.com

${ }^{2}$ Doutores em Economia. Professores da Universidade das Ilhas Baleares.

* Os autores reconhecem e agradecem a contribuição do professor Wilson Abrahão Rabahy para o desenvolvimento deste trabalho.
} 
this uncommon Brazilian reality: changes in the behavior of tourists visiting Brazil, and changes in the composition of the Brazilian inbound tourism flow. The endorsement of the first hypothesis would show that the evolution of tourist behavior over the last years was actually different in Brazil and in the rest of the world. This would evidence the occurrence of improvements in the quality of tourist experiences offered by Brazil. The present study aims to test this hypothesis by using duration models to analyze more than 211 thousand tourist surveys. Modeling individual tourist behavior allows to control for intervenient variables and to conduct a detailed analysis of the time effect on the expected tourists' length of stay. Thirteen duration models are tested. The model with Weibull distributed hazard and Gamma distributed individual heterogeneity is selected. The estimates show that the increasing trend of tourists' length of stay in Brazil is partially explained by changes in tourists' behavior.

Keywords: Length of stay; Duration of the trip; Duration models; Tourism in Brazil.

Resumen: En todo el mundo la duración media de las viajes se ha reducido. Mientras, curiosamente, esa tendencia no se ha observado en el turismo internacional receptivo de Brasil. Dos hipótesis pueden explicar la distinta realidad brasileña: cambios en el comportamiento de los turistas que visitan el país, y cambios en la composición del flujo turístico receptivo internacional brasileño. Una eventual confirmación de la primera hipótesis comprobaría que la evolución de lo comportamiento de los turistas en los últimos años fue efectivamente diferente en Brasil y en los otros países, evidenciando mejoramientos en la calidad de la experiencia turística ofertada por Brasil. Ese estudio testa esa hipótesis por medio del uso de modelos de duración basados en más de 211 mil encuestas con turistas. La modelización del comportamiento individual de los turistas permite controlar el efecto de las variables intervinientes, posibilitando una evaluación detallada del efecto temporal sobre la duración esperada de las estancias de los turistas. Trece modelos de duración son testados. El modelo con mejor ajuste a los datos tiene tasa de riesgo con distribución Weibull y heterogeneidad individual con distribución Gama. Los resultados apuntan que la tendencia creciente de la permanencia media es parcialmente explicada por un cambio en el comportamiento de los turistas.

Palabras clave: Permanencia; Duración; Estancia; Modelos de duración; Turismo en Brasil.

\section{INTRODUÇÃO}

De acordo com a Organização Mundial do Turismo, duração média das viagens no mundo tem diminuído (UNWTO, 2006, 2007). Segundo essa entidade, as viagens de curta duração têm se tornado uma alternativa cada vez mais popular. A tendência mundial decrescente também é confirmada por diversos estudos acadêmicos (Alegre \& Pou, 2006; Barros, Correia, \& Crouch, 2008; Barros \& Machado, 2010; Martínez-Garcia \& Raya, 2008). No entanto, curiosamente, essa tendência não tem se revelado no Brasil. De acordo com dados do Ministério do Turismo, a permanência média dos turistas internacionais no Brasil entre 1993 e 2010 cresceu aproximadamente $1,6 \%$ ao ano.

Essa taxa de crescimento não foi constante. Entre 1993 e 2003 a permanência média se manteve praticamente estável, evoluindo de 13,1 para 13,5 dias. Já entre 2003 e 2007 a permanência média cresceu quase 40\%, atingindo um pico de 18,8 dias em 2006 e 2007. Nos anos seguintes a permanência média caiu quase $10 \%$, atingindo 17,2 dias em 2010 . A evolução da permanência média dos turistas internacionais no Brasil é apresentada na Figura 1. 
Figura 1. Evolução da permanência media dos turistas internacionais no Brasil

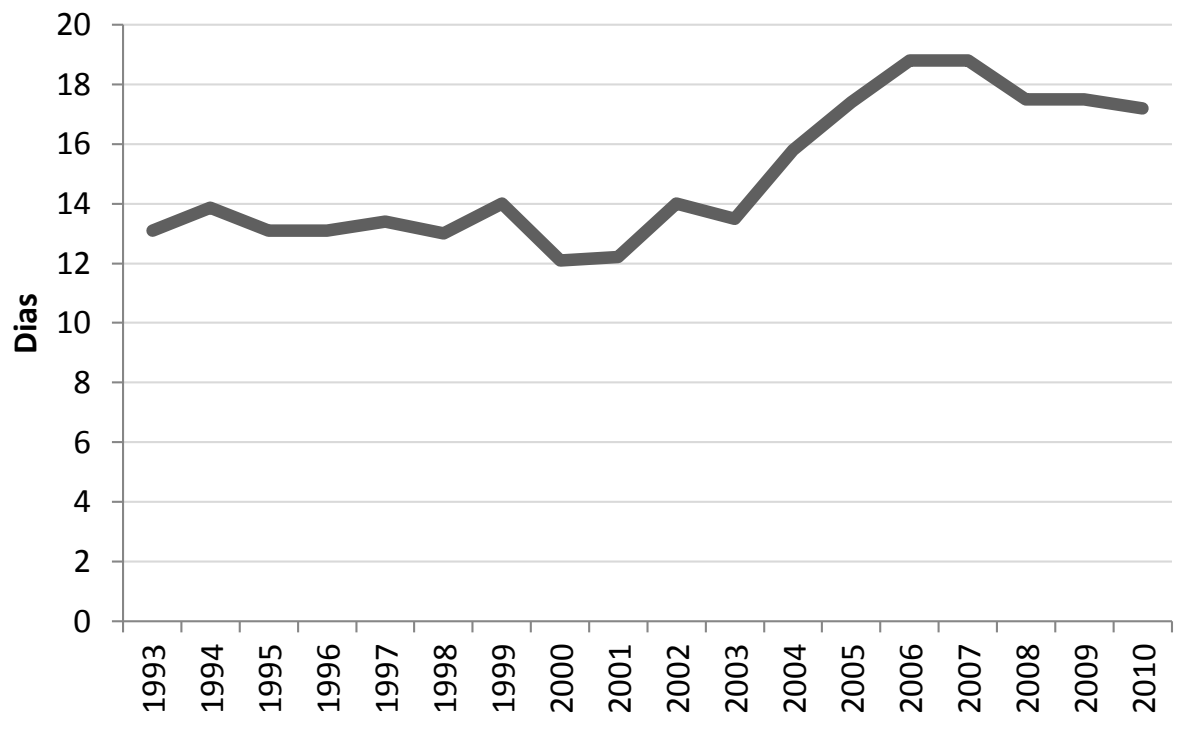

Fonte: Ministério do Turismo

O incomum aumento da permanência média dos turistas ocorrido no Brasil pode ter duas explicações distintas. A primeira é o aumento da duração esperada da estada de turistas independentemente de suas características. Por exemplo, se essa explicação fosse verdadeira, um determinado indivíduo que tivesse vindo ao Brasil em 2010 tenderia a ficar no país por um período de tempo maior do que se tivesse vindo alguns anos antes. Uma eventual confirmação desta primeira explicação mostraria que o fluxo turístico receptivo internacional no Brasil está efetivamente evoluindo em um sentido diferente do que ocorre no resto do mundo. Tal confirmação indicaria que, no período analisado, o Brasil desenvolveu uma maior capacidade de retenção dos turistas em seu território. Portanto, essa explicação está associada à melhoria da oferta de experiências aos turistas que visitam o país.

Uma explicação alternativa para o aumento da permanência média consiste na alteração da composição do fluxo turístico receptivo internacional brasileiro. Permanências médias maiores podem ser fruto da atração de turistas com perfis diferenciados e tendência a ficarem no destino por períodos maiores. Um exemplo prático disto seria a atração de turistas residentes em países mais distantes, já que distância e tempo de permanência usualmente apresentam correlação positiva. A explicação da realidade brasileira por essa via significaria que, no período analisado, o país passou a atrair proporcionalmente mais turistas com preferências por estadas longas. Enquanto a primeira explicação está associada à oferta de experiências melhores aos turistas que selecionam o Brasil como destino, a segunda diz respeito à própria 
seleção do país como destino da viagem. Essas duas hipóteses explicativas são sintetizadas a seguir.

1) O aumento do tempo médio de permanência dos turistas internacionais no Brasil é explicado por mudanças no comportamento dos turistas.

2) O aumento do tempo médio de permanência dos turistas internacionais no Brasil é fruto de alterações na composição do fluxo turístico receptivo internacional brasileiro.

A segunda explicação é facilmente verificada por meio de algumas análises estatísticas simples. Por exemplo, em 1993 aproximadamente 67\% do fluxo turístico internacional receptivo no Brasil eram compostos por sul americanos. Em 2010 essa fatia havia caído para 46\%. Como os sul-americanos em média ficam períodos menores no Brasil, o aumento da permanência média é explicado, ao menos parcialmente, pela queda na participação desse público no fluxo total.

No entanto, a verificação da segunda hipótese não implica na rejeição da primeira, já que as duas explicações podem ser concomitantemente verdadeiras. No entanto, o teste da primeira hipótese exige o emprego de técnicas estatísticas mais sofisticadas, uma vez que o comportamento dos turistas é influenciado por diversas variáveis. Neste sentido, o presente estudo utiliza modelos de duração a fim de explicar o tempo de permanência dos turistas internacionais no Brasil a partir da tendência temporal e das características dos turistas e de suas viagens. Esse procedimento permite desagregar os efeitos de cada variável explicativa, possibilitando uma avaliação detalhada do efeito temporal sobre a duração esperada das estadas dos turistas independentemente de suas características. O estudo é baseado em informações obtidas por meio de 211 mil entrevistas com turistas estrangeiros realizadas entre 2004 e 2011 no âmbito da pesquisa de Caracterização e Dimensionamento do Turismo Internacional no Brasil realizada pela Fundação Instituto de Pesquisas Econômicas (FIPE) e parceria com o Ministério do Turismo.

\section{FUNDAMENTAÇÃO TEÓRICA}

O interesse acadêmico na duração da estada dos turistas é relativamente antigo. Wurst (1955), um pioneiro nesta área, propôs uma forma de cálculo da permanência média a partir de dados agregados. Duas décadas mais tarde, Archer e Shea (1975) buscaram aprofundar a discussão sobre esse assunto. Diferentes objetivos e perspectivas sobre o tema foram adotados por estudos mais recentes. Análises descritivas e univariadas da permanência dos turistas e das variáveis determinantes desse comportamento foram desenvolvidas por Oppermann (1994, 1995, 1997), Seaton e Palmer (1997), Sung, Morrison, Hong e O'Leary (2001) e Tierney (1993).

Análises multivariadas dos determinantes da permanência dos turistas foram realizadas por 21 estudos baseados em microdados. Uma síntese das características desses estudos é apresentada na Tabela 1. 
Santos, G. E. de O., Ramos, V., \& Rey-Maquieira, J. Aumento do tempo de permanência dos turistas no Brasil: Novos turistas ou novos comportamentos?

Tabela 1. Síntese dos estudos sobre o tempo de permanência dos turistas

\begin{tabular}{|c|c|c|c|c|c|}
\hline Estudo & Destino analisado & $\begin{array}{c}\text { Tamanho da } \\
\text { amostra }\end{array}$ & Modelo & $\begin{array}{c}\text { Distribuições estatísticas } \\
\text { ou formas funcionais }\end{array}$ & $\begin{array}{l}\text { Características } \\
\text { especiais }\end{array}$ \\
\hline $\begin{array}{l}\text { Mak, Moncur, e } \\
\text { Yonamine (1977) }\end{array}$ & Havaí, EUA & 4.990 & MQME & linear & Dois estágios \\
\hline Mak e Nishimura (1979) & Havaí, EUA & 690 & MQO & $\log -\log$ & \\
\hline Walsh e Davitt (1983) & Aspen, EUA & 837 & MQO & $\begin{array}{l}\text { linear, quadrática, log- } \\
\text { linear*, log-log }\end{array}$ & \\
\hline Silberman (1985) & $\begin{array}{l}\text { Virginia Beach, } \\
\text { EUA }\end{array}$ & 621 & MQME & $\begin{array}{l}\text { linear, quadrática, log- } \\
\text { linear, log-log* }\end{array}$ & Dois estágios \\
\hline $\begin{array}{l}\text { Uysal, McDonald, e } \\
\text { O'Leary (1988) }\end{array}$ & USA & 6.720 & $\begin{array}{l}\text { MQO, } \\
\text { MQME* }\end{array}$ & $\operatorname{lin}-\log$ & Dois estágios \\
\hline Paul e Rimmawi (1992) & $\begin{array}{l}\text { Parque Nacional } \\
\text { Asir, Arábia } \\
\text { Saudita }\end{array}$ & 208 & MQO & linear & \\
\hline $\begin{array}{c}\text { Blaine, Mohammad, e } \\
\operatorname{Var}(1993)\end{array}$ & $\begin{array}{c}\text { Fredericksburg, } \\
\text { EUA }\end{array}$ & - & MQO & $\log -\log$ & \\
\hline Alegre e Pou (2006) & $\begin{array}{c}\text { Ilhas Baleares, } \\
\text { Espanha }\end{array}$ & 24.896 & Binário & logit & \\
\hline Hellström (2006) & $\begin{array}{l}\text { Estocolmo, } \\
\text { Gotemburgo e } \\
\text { Malmö, Suécia }\end{array}$ & 2.000 & Contagem & $\begin{array}{l}\text { Poisson log-normal } \\
\text { bivariado }\end{array}$ & Inflação, Truncagem \\
\hline $\begin{array}{c}\text { Gokovali, Bahar, e Kozak } \\
\text { (2007) }\end{array}$ & Bodrum, Turquia & 672 & Duração & $\begin{array}{l}\text { Cox*, exponencial, } \\
\text { Weibull*, Gompertz }\end{array}$ & \\
\hline Barros et al. (2008) & América Latina & 442 & Duração & $\begin{array}{l}\text { Cox, Weibull**, log- } \\
\text { logística }\end{array}$ & Heterogeneidade \\
\hline $\begin{array}{c}\text { Martínez-Garcia e Raya } \\
\text { (2008) }\end{array}$ & $\begin{array}{l}\text { Catalunha, } \\
\text { Espanha }\end{array}$ & 990 & Duração & $\begin{array}{c}\text { Cox, exponencial, } \\
\text { Weibull, Gompertz, log- } \\
\text { normal, log-logística*, } \\
\text { Gamma }\end{array}$ & Heterogeneidade \\
\hline $\begin{array}{l}\text { Menezes, Moniz, e Vieira } \\
\text { (2008) }\end{array}$ & Açores, Portugal & 400 & Duração & Cox & \\
\hline $\begin{array}{c}\text { Barros, Butler e Correia } \\
(2010)\end{array}$ & Algarve, Portugal & 593 & Duração & Cox, Weibull** & $\begin{array}{l}\text { Heterogeneidade, } \\
\text { seleção amostral }\end{array}$ \\
\hline Barros e Machado (2010) & $\begin{array}{l}\text { Madeira, } \\
\text { Portugal }\end{array}$ & 346 & Duração & Weibull & Seleção amostral \\
\hline Machado (2010) & $\begin{array}{l}\text { Madeira, } \\
\text { Portugal }\end{array}$ & 346 & Duração & Weibull & $\begin{array}{l}\text { Seemingly unrelated } \\
\text { discrete-choice } \\
\text { duration }\end{array}$ \\
\hline $\begin{array}{l}\text { Alegre, Mateo e Pou } \\
\text { (2011) }\end{array}$ & $\begin{array}{l}\text { Ilhas Baleares, } \\
\text { Espanha }\end{array}$ & 29.162 & Contagem & Poisson & Class latente \\
\hline Raya e Martínez-Garcia & $\begin{array}{l}\text { Catalunha, } \\
\text { Espanha }\end{array}$ & - & Duração & $\begin{array}{c}\text { Cox, exponencial, } \\
\text { Weibull, Gompertz, log- } \\
\text { normal, log-logística*, } \\
\text { Gamma }\end{array}$ & Heterogeneidade \\
\hline $\begin{array}{c}\text { Yang, Wong e Zhang } \\
(2011)\end{array}$ & Yixing, China & 417 & Ordinal & logit & \\
\hline $\begin{array}{c}\text { Peypoch, } \\
\text { Reriamboarison, } \\
\text { Rasoamananjara e } \\
\text { Solonerasana (2012) }\end{array}$ & Madagascar & 615 & Duração & $\begin{array}{l}\text { Polinomial fracional, } \\
\text { Weibull }\end{array}$ & \\
\hline
\end{tabular}

Fonte: Elaboração própria. 
A dimensão das amostras utilizadas nos estudos anteriores é substancialmente heterogênea. Muitos estudos utilizaram amostras relativamente pequenas, especialmente se comparadas ao grande número de parâmetros dos modelos, o que frequentemente levou à estimação de inúmeros parâmetros não significantes. Duas exceções dignas de nota são os estudos de Alegre et al. (2011) e Alegre e Pou (2006).

Diferentes métodos estatísticos foram utilizados nos estudos anteriores, incluindo Mínimos Quadrados Ordinários (MQO), Mínimos Quadrados em Múltiplos Estágios (MQME), logit binário, logit ordinal, regressão de Poisson truncada de classe latente, regressão de Poisson bivariada log-normal e diversos modelos de duração. Em particular, os modelos de duração constituem a alternativa mais adequada para a modelagem do tempo de estada dos turistas uma vez que garantem a positividade da variável dependente e oferecem inúmeras alternativas de distribuição do erro estocástico. Esses modelos foram apenas recentemente aplicados ao estudo da duração da permanência dos turistas (Gokovali et al., 2007), mas sua popularidade cresceu rapidamente. Nos estudos sobre o tempo de permanência dos turistas, diferentes modelos de duração foram utilizados, incluindo versões semi-paramétricas e paramétricas. Dentre as versões paramétricas, os modelos mais frequentes são exponencial, Weibull, Gompertz, log-normal, log-logística e Gama. Além disso, diversos estudos utilizaram modelos de duração com características especiais, tais como heterogeneidade e controle para o problema da seleção amostral.

Os efeitos de inúmeras variáveis sobre o tempo de permanência dos turistas têm sido estudados. A maior parte dos estudos estimou que gênero não é uma variável significante (Barros et al., 2010; Machado, 2010; Martínez-Garcia \& Raya, 2008; Menezes et al., 2008; Raya \& Martínez-Garcia, 2011), ainda que Barros e Machado (2010) e Peypoch et al. (2012) tenham estimado que homens tendem a ficar no destino por períodos mais longos. Alguns estudos estimaram que a idade não é um determinante relevante do tempo de permanência dos turistas (Gokovali et al., 2007; Menezes et al., 2008; Raya \& Martínez-Garcia, 2011), ao passo que Barros et al. (2008) encontrou uma relação negativa entre idade e permanência. No entanto, a maior parte dos estudos aponta que o tempo de permanência cresce com o aumento da idade dos turistas (Alegre \& Pou, 2006; Barros et al., 2010; Barros \& Machado, 2010; Hellström, 2006; Machado, 2010; Mak et al., 1977; Martínez-Garcia \& Raya, 2008; Peypoch et al., 2012). Finalmente, Yang, Wong e Zhang (2011) estimam que a permanência esperada diminui com o aumento da idade até cerca de 35 anos, ponto a partir do qual a relação entre ambas as variáveis se torna positiva.

Os estudos anteriores não são conclusivos com respeito à relação entre escolaridade e tempo de permanência dos turistas. Três estudos estimaram que maiores níveis de escolaridade resultam em estadas mais curtas (Gokovali et al., 2007; Martínez-Garcia \& Raya, 2008; Menezes et al., 2008), enquanto três outros estudos estimaram o contrário (Barros et al., 2010; Barros \& Machado, 2010; Peypoch et al., 2012). Por fim, dois estudos não encontraram nenhuma relação entre ambas as variáveis (Machado, 2010; Raya \& Martínez-Garcia, 2011). 
A maior parte dos estudos estima que o tempo de permanência no destino é um bem normal, isto é, maiores níveis de renda levam a permanências mais longas (Barros et al., 2008; Gokovali et al., 2007; Mak et al., 1977; Peypoch et al., 2012; Silberman, 1985; Walsh \& Davitt, 1983). No entanto, Hellström (2006) e Barros et al. (2010) não encontraram evidências de qualquer relação entre renda e permanência. Além disso, Blaine et al. (1993) e Mak e Nishimura (1979) encontraram evidências de uma relação negativa entre ambas as variáveis, indicando o tempo de estada como um bem inferior.

O efeito da nacionalidade dos turistas foi analisado por dez estudos (Alegre et al., 2011; Alegre \& Pou, 2006; Barros et al., 2010; Barros \& Machado, 2010; Gokovali et al., 2007; Machado, 2010; Martínez-Garcia \& Raya, 2008; Menezes et al., 2008; Peypoch et al., 2012; Raya \& Martínez-Garcia, 2011). Os parâmetros estimados pela maior parte foram significativos, indicando que o tempo esperado de permanência dos turistas varia de acordo com o mercado de origem. Contudo, nenhum padrão claro foi encontrado com respeito às características dos países associados a estadas mais longas.

O motivo da viagem é uma variável fundamental na explicação do tempo de permanência dos turistas no destino. A maior parte dos estudos anteriores analisaram os efeitos dessa variável, sendo que resultados não significantes foram obtidos apenas por Martínez-Garcia e Raya (2008) e Raya e Martínez-Garcia (2011). As evidências empíricas mostram que o efeito de motivações específicas varia de acordo com o destino analisado.

Aqui foram destacadas as variáveis explicativas que serão utilizadas neste estudo. No entanto, além destas, estudos anteriores analisaram os efeitos de diversas outras características dos indivíduos, tais como ocupação (Alegre \& Pou, 2006; Alegre et al., 2011; Blaine et al., 1993; Gokovali et al., 2007; Martínez-Garcia \& Raya, 2008; Raya \& Martínez-Garcia, 2011; Menezes et al., 2008), estado civil (Barros et al., 2008; Gokovali et al., 2007; Mak et al., 1977; MartínezGarcia \& Raya, 2008; Menezes et al., 2008; Raya \& Martínez-Garcia, 2011), comportamento de viagem (Alegre et al., 2011; Alegre \& Pou, 2006; Barros et al., 2008; Gokovali et al., 2007; Uysal et al., 1988), composição familiar (Hellström, 2006), e atitudes relativas à iniciativas sustentáveis (Menezes et al., 2008). Os efeitos de diversas características das viagens também foram analisados por estudos anteriores. No entanto, o tratamento dessas variáveis explicativas é delicado na medida em que pode incorrer em problemas de endogeneidade.

\section{FONTE DE INFORMAÇÕES E VARIÁVEIS}

A análise do tempo de permanência dos turistas estrangeiros no Brasil foi realizada com base em dados da pesquisa de Caracterização e Dimensionamento do Turismo Internacional no Brasil, projeto que visa levantar dados e gerar estatísticas oficiais sobre as características do fluxo turístico receptivo do Brasil a fim de prover informações úteis para a gestão pública e privada do turismo no país. É com base nos dados dessa pesquisa que o Ministério do Turismo tem estimado a permanência média dos turistas no Brasil desde 2004. O levantamento de dados 
é realizado por meio de entrevistas diretas com turistas nos 27 principais portões de saída do Brasil. Os 15 aeroportos internacionais ${ }^{3}$ incluídos na pesquisa respondem por cerca de $100 \%$ do fluxo aéreo internacional de passageiros no país. Já o fluxo terrestre de turistas é mais disperso, sendo que as 12 fronteiras terrestres ${ }^{4}$ pesquisadas respondem por cerca de $90 \%$ do total no país. A coleta de dados ocorre em quatro etapas anuais realizadas nos meses de Janeiro, Abril, Julho e Outubro. As entrevistas são conduzidas em seis diferentes idiomas, a saber: Português, Inglês, Espanhol, Francês, Italiano e Alemão. Desse banco de dados foram utilizadas informações de 211.371 entrevistas realizadas entre 2004 e 2011.

A variável dependente analisada neste estudo é o tempo de permanência do turista no Brasil medido em pernoites. Por convenção essa variável tem valor mínimo um e máximo 365. Sete conjuntos de variáveis foram utilizados para explicar o tempo de permanência individual. A variável idade é numérica, enquanto o gênero é representado por uma variável dummy. O nível de escolaridade do entrevistado foi classificado em quatro categorias: sem ensino médio completo, ensino médio completo, ensino superior completo e pós-graduação completa. O logaritmo da renda familiar medida em dólares constantes de 2010 também foi inserido no modelo. O uso do logaritmo justifica-se pela assimetria positiva da distribuição dessa variável explicativa. O país de origem do entrevistado também foi utilizado como variável explicativa. Os onze países que emitem anualmente mais de 100 mil turistas para o Brasil foram identificados por variáveis dummy específicas. Os demais países foram agrupados por continentes. Outra variável explicativa utilizada foi o motivo da viagem, a qual foi classificada em sete categorias: sol e praia, ecoturismo, turismo cultural, outros motivos de lazer, negócios, visita a amigos e parentes, e outros motivos de não-lazer.

Por fim, o ano de realização da entrevista constitui uma variável explicativa destinada a captar a evolução da permanência esperada dos turistas. A estimativa de um coeficiente significativo indicando maiores permanências para os anos mais recentes comprovaria a hipótese 1 de que o aumento do tempo médio de permanência dos turistas internacionais no Brasil se deve a uma mudança no comportamento dos turistas. A estimativa de um coeficiente indicando permanências menores para anos mais recentes, ou a estimativa de um coeficiente não-significativo, levaria à rejeição da hipótese 1.

\section{MÉTODO E RESULTADOS}

Os modelos de duração são construídos para modelar o lapso de tempo entre o início e o término de qualquer fenômeno, sendo frequentemente utilizados nas áreas da medicina, engenharia e economia. Neste estudo, essa classe de modelos é utilizada para a análise do

\footnotetext{
${ }^{3}$ Belém (PA), Belo Horizonte (MG), Brasília (DF), Curitiba (PR), Florianópolis (SC), Fortaleza (CE), Maceió (AL), Manaus (AM), Natal (RN), Porto Alegre (RS), Porto Seguro (BA), Recife (PE), Rio de Janeiro (RJ), Salvador (BA), São Paulo (SP).

${ }^{4}$ Chuí (RS), Corumbá (MS), Dionísio Cerqueira (SC), Epitaciolândia (AC), Foz do Iguaçu (PR) - Ponte Internacional da Amizade e Ponte Tancredo Neves, Jaguarão (RS), Pacaraima (RR), Ponta Porã (MS), Santana do Livramento (RS), São Borja (RS), Uruguaiana (RS).
} 
tempo de permanência dos turistas estrangeiros no Brasil. Para obter informações detalhadas sobre esses modelos, o leitor é convidado aos trabalhos de Hougaard (1999), Kiefer (1988) e Lancaster (1990).

Duas funções principais são analisadas através dos modelos de duração. A função de sobrevivência indica a probabilidade de que o fenômeno dure mais do que um determinado valor de tempo (t). Aplicado ao turismo, a função de sobrevivência se refere à probabilidade de que o turista permaneça no destino mais do que $t$ dias. A taxa de risco indica a probabilidade de que o fenômeno termine no próximo período dado que ele durou até o momento atual.

A duração de um fenômeno pode ser condicionada por uma série de variáveis explicativas (x). Por exemplo, o tempo de permanência do turista no destino pode ser influenciado por sua renda, idade e gostos. Existem duas abordagens paramétricas para introduzir $\mathbf{x}$ no modelo de duração: risco proporcional e tempo de vida acelerado. Diferentes parametrizações da duração resultam em diferentes modelos. As versões mais comuns dos modelos de risco proporcional são a exponencial, Weibull e Gompertz. Dentre os modelos de tempo de vida acelerado destacam-se as versões exponencial, log-normal, log-logística e Gama. Na distribuição exponencial, a qual é compatível com ambas as abordagens para a introdução $\mathbf{x}$, a taxa de risco é constante. No caso das distribuições Weibull e Gompertz a taxa de risco segue uma função monotônica do tempo. Já nas distribuições log-normal e log-logística a taxa de risco segue distribuições com assimetria positiva. Por fim, a taxa de risco na distribuição Gama pode tanto ser monotonicamente decrescente quanto positivamente assimétrica.

Outra opção para a introdução das variáveis explicativas nos modelos de duração é a semi-paramétrica. Uma vez que a forma funcional da taxa de risco é usualmente desconhecida $a$ priori, e que proposições teóricas sobre essa distribuição são escassas, deixar a taxa de risco sem especificação pode evitar arbitrariedades. O resultado dessa abordagem é o modelo semiparamétrico de risco proporcional de Cox (1972). No presente estudo esse modelo foi descartado em virtude da rejeição da hipótese de risco proporcional segundo o teste Schoenfeld (Grambsch \& Therneau, 1994; Schoenfeld, 1982).

Heterogeneidade individual não-observada nos modelos de duração constitui uma preocupação especial. Esse tipo de heterogeneidade pode ser resultado de erros de medida ou da omissão de variáveis explicativas relevantes (Lancaster, 1979; Vaupel, Manton, \& Stallard, 1979). De acordo com Heckman e Singer (1984), sempre que a heterogeneidade em dados de duração é testada em estudos microeconômicos o resultado é positivo. A omissão da heterogeneidade não-observada pode distorcer as estimativas do modelo (Lancaster, 1979). Portanto, incluir heterogeneidade nos modelos de duração é um requisito fundamental para a obtenção de estimativas confiáveis. Para tanto, duas distribuições do termo de heterogeneidade têm sido comumente aplicadas: Gama e Normal Invertida (Hougaard, 1984).

O presente estudo estimou modelos paramétricos de duração utilizando todas as seis alternativas de distribuição da duração combinadas com as duas alternativas de distribuição da heterogeneidade individual aqui discutidas. Logo, além do modelo semi-paramétrico de risco 
proporcional de Cox, foram estimados doze modelos dentre os quais o melhor foi selecionado de acordo com o critério de informação de Akaike e com as estatísticas de log-verossimilhança e qui-quadrado. As três estatísticas apontaram que o modelo que melhor se ajusta aos dados é aquele cuja taxa de risco segue uma distribuição Weibull e a heterogeneidade individual segue uma distribuição Gama. A função de sobrevivência nesse modelo é dada por

$$
F(t)=\left(e^{-e^{-p x \beta} t^{p}}\right)^{\alpha}
$$

onde t se refere ao tempo, p é um parâmetro referente à distribuição Weibull da taxa de risco, $\mathbf{x}$ é um vetor de variáveis independentes, $\boldsymbol{\beta}$ é um vetor de parâmetros associados às variáveis independentes, e $\alpha$ é o parâmetro referente à heterogeneidade observada com média igual a 1 e variância igual a $\theta$.

As estimativas obtidas a partir da aplicação do modelo selecionado ao conjunto de dados são apresentadas na Tabela 2. Os coeficientes seguem a métrica de tempo de vida acelerado, de forma que coeficientes positivos indicam que um aumento no valor da variável independente causa uma ampliação no tempo esperado de permanência dos turistas internacionais no Brasil. Além dos coeficientes estimados, apresentam-se também o desvio-padrão de cada coeficiente e o p-valor. Na primeira linha das variáveis nível de escolaridade, país de residência e motivo da viagem são apresentados os $\mathrm{p}$-valores do teste qui-quadrado para a hipótese de que todos os coeficientes dessas variáveis multinominais são concomitantemente iguais a zero.

Tabela 2. Estimativas do modelo selecionado

\begin{tabular}{|c|c|c|c|}
\hline Variável & Coeficiente & Desvio-padrão & $\mathrm{p}$-valor \\
\hline Idade & $-0,0030$ & 0,00014 & 0,00 \\
\hline \multicolumn{4}{|l|}{ Gênero } \\
\hline Feminino* & 0,00 & & \\
\hline Masculino & $-0,11$ & 0,0038 & 0,00 \\
\hline Nível de escolaridade & & & 0,00 \\
\hline Sem ensino médio completo* & 0,00 & & \\
\hline Ensino médio completo & 0,20 & 0,0091 & 0,00 \\
\hline Ensino superior completo & 0,071 & 0,0091 & 0,00 \\
\hline Pós-graduação completa & $-0,018$ & 0,010 & 0,057 \\
\hline Renda & $-0,0054$ & 0,0014 & 0,00 \\
\hline País de residência & & & 0,00 \\
\hline \multicolumn{4}{|l|}{ África } \\
\hline Todos os países* & 0,00 & & \\
\hline \multicolumn{4}{|l|}{ América Central } \\
\hline Todos os países & $-0,20$ & 0,024 & 0,00 \\
\hline \multicolumn{4}{|l|}{ América do Norte } \\
\hline Estados Unidos & $-0,16$ & 0,015 & 0,00 \\
\hline Outros países & $-0,080$ & 0,017 & 0,00 \\
\hline \multicolumn{4}{|l|}{ América do Sul } \\
\hline Argentina & $-0,40$ & 0,015 & 0,00 \\
\hline Chile & $-0,55$ & 0,016 & 0,00 \\
\hline Paraguai & $-1,3$ & 0,017 & 0,00 \\
\hline Uruguai & $-0,75$ & 0,018 & 0,00 \\
\hline
\end{tabular}


Santos, G. E. de O., Ramos, V., \& Rey-Maquieira, J. Aumento do tempo de permanência dos turistas no Brasil: Novos turistas ou novos comportamentos?

\begin{tabular}{|c|c|c|c|}
\hline Outros países & $-0,37$ & 0,016 & 0,00 \\
\hline \multicolumn{4}{|l|}{ Ásia e Oceania } \\
\hline Todos os países & 0,17 & 0,017 & 0,00 \\
\hline \multicolumn{4}{|l|}{ Europa } \\
\hline Alemanha & 0,15 & 0,016 & 0,00 \\
\hline Espanha & 0,049 & 0,016 & 0,0030 \\
\hline França & 0,078 & 0,016 & 0,00 \\
\hline Inglaterra & $-0,00080$ & 0,017 & 1,0 \\
\hline Itália & 0,19 & 0,016 & 0,00 \\
\hline Portugal & $-0,021$ & 0,016 & 0,18 \\
\hline Outros países & 0,13 & 0,015 & 0,00 \\
\hline Motivo da viagem & & & 0,00 \\
\hline \multicolumn{4}{|l|}{ Lazer } \\
\hline Sol e praia* & 0,00 & & \\
\hline Ecoturismo & $-0,42$ & 0,0071 & $-58,6$ \\
\hline Turismo cultural & $-0,29$ & 0,0082 & $-35,7$ \\
\hline Outros motivos de lazer & $-0,34$ & 0,011 & $-31,5$ \\
\hline \multicolumn{4}{|l|}{ Não-lazer } \\
\hline Negócios & $-0,68$ & 0,0053 & $-128,7$ \\
\hline Visita a amigos e parentes & 0,23 & 0,0052 & 43,4 \\
\hline Outros motivos de não-lazer & 0,10 & 0,011 & 8,6 \\
\hline Ano & 0,012 & 0,0011 & 0,00 \\
\hline \multicolumn{4}{|l|}{ Constantes } \\
\hline$\beta_{0}$ & 2,7 & 0,018 & 0,00 \\
\hline$p$ & 2,4 & 0,0090 & 0,00 \\
\hline$\theta$ & 1,3 & 0,010 & 0,00 \\
\hline
\end{tabular}

\section{DISCUSSÃO E CONSIDERAÇÕES FINAIS}

A significância da variância do parâmetro $\alpha$ dada por $\theta$ indica que a heterogeneidade individual não-observada é relevante e deve ser incluída no modelo. Logo, modelos de duração sem heterogeneidade são inadequados para a modelagem do tempo de permanência dos turistas, oferecendo estimativas visadas.

Com respeito à idade e gênero, os resultados apontam que o tempo de permanência esperado é maior para turistas mais jovens e do sexo feminino. O efeito da variável nível de escolaridade é significante, mas não apresenta um padrão muito claro. Turistas sem ensino médio completo tendem a ficar por períodos de tempo mais curtos no Brasil do que aqueles que completaram essa etapa de escolaridade. Logo, nesse trecho da escala, maiores permanências estão associadas a maiores níveis de escolaridade. Contudo, a partir do ensino médio essa relação se inverte. Turistas com ensino superior completo tendem a ficar no Brasil por períodos menores do que aqueles que têm apenas ensino médio completo. Na comparação entre ensino superior e pós-graduação, novamente o aumento do nível de escolaridade está associado a uma redução no tempo esperado de permanência.

O tempo de permanência no Brasil é tido como um bem inferior pelos turistas internacionais. Quanto maior a renda familiar, menor é a permanência esperada do turista no país. Essa relação é relativamente incomum, sendo conflitante com as evidências empíricas 
proporcionadas pela maior parte dos estudos anteriores (Barros et al., 2008; Gokovali et al., 2007; Mak et al., 1977; Peypoch et al., 2012; Silberman, 1985; Walsh \& Davitt, 1983). No entanto, a inferioridade é consistente com as evidências encontradas por Blaine et al. (1993) e Mak e Nishimura (1979). A explicação detalhada dessa relação negativa entre renda e permanência pode constituir um problema de pesquisa bastante interessante para trabalhos futuros.

Visitas a amigos e parentes é a motivação mais fortemente associada às longas permanências. Dentre os motivos identificados, sol e praia ocupa a segunda colocação nesse ranking. Na sequência tem-se o turismo cultural e o ecoturismo, sendo que os negócios constituem a motivação mais fortemente associada às permanências curtas no Brasil.

Por fim, cabe analisar as estimativas referentes à variável ano. O coeficiente estimado para essa variável é positivo e significante, indicando que o tempo de permanência esperado de turistas com mesmas características apresentou tendência crescente entre 2004 e 2011. Portanto, aceita-se a hipótese 1 , ou seja, confirma-se que o aumento no tempo médio de permanência dos turistas internacionais no Brasil não é fruto exclusivo de alterações na composição do fluxo turístico receptivo internacional brasileiro, mas também de mudanças no comportamento dos turistas. Essa realidade diverge da tendência mundial e indica uma situação privilegiada do Brasil. O aumento da permanência dos turistas com características constantes aponta para melhorias na qualidade da experiência turística ofertada pelo Brasil. 0 desenvolvimento da oferta de uma experiência turística de qualidade crescente, cada vez mais capaz de reter os turistas por mais tempo, pode ter diferentes causas, incluindo principalmente a diversificação da oferta de atrativos, melhorias na qualidade dos serviços e a facilitação do acesso a diferentes destinos dentro do país. A detalhada análise das causas dessa mudança no comportamento dos turistas constitui um tema de grande importância a ser desenvolvido por estudos futuros.

\section{REFERÊNCIAS}

Alegre, J., Mateo, S., \& Pou, L. (2011). A latent class approach to tourists' length of stay. Tourism Management, 32(3), 555-563.

Alegre, J., \& Pou, L. (2006). The length of stay in the demand for tourism. Tourism Management, 27(6), 1343-1355.

Archer, B. H., \& Shea, S. (1975). Length of stay problems in tourist research. Journal of Travel Research, 13(3), 8-10.

Barros, C. P., Butler, R., \& Correia, A. (2010). The length of stay of golf tourism: A survival analysis. Tourism Management, 31(1), 13-21.

Barros, C. P., Correia, A., \& Crouch, G. (2008). Determinants of the length of stay in Latin American tourism destinations. Tourism Analysis, 13(4), 329-340.

Barros, C. P., \& Machado, L. P. (2010). The length of stay in tourism. Annals of Tourism Research, 37(3), 692-706. 
Blaine, T. W., Mohammad, G., \& Var, T. (1993). Demand for rural tourism: an exploratory study. Annals of Tourism Research, 20(4), 770-773.

Cox, D. R. (1972). Regression models and life-tables. Journal of the Royal Statistical Society (Series B), 43(2), 187-220.

Gokovali, U., Bahar, O., \& Kozak, M. (2007). Determinants of length of stay: a practical use of survival analysis. Tourism Management, 28(3), 736-746.

Grambsch, P. M., \& Therneau, T. M. (1994). Proportional hazards tests and diagnostics based on weighted residuals. Biometrika, 81, 515-526.

Heckman, J., \& Singer, B. (1984). A method for minimizing the impact of distributional assumptions in econometric models for duration data. Econometrica, 52(2), 271-320.

Hellström, J. (2006). A bivariate count data model for household tourism demand. Journal of Applied Econometrics, 21(2), 213-226.

Hougaard, P. (1984). Life table methods for heterogeneous populations: distributions describing the heterogeneity. Biometrika, 71(1), 75-83.

Hougaard, P. (1999). Fundamentals of survival data. Biometrics, 55(1), 13-22.

Kiefer, N. M. (1988). Economic duration data and hazard functions. Journal of Economic Literature, 26(2), 646-679.

Lancaster, T. (1979). Econometric methods for the duration of unemployment. Econometrica, 47(4), 939956.

Lancaster, T. (1990). The Analysis of Transition Data. New York: Cambridge University Press.

Machado, L. P. (2010). Does destination image influence the length of stay in a tourism destination? Tourism Economics, 16(2), 443-456.

Mak, J., Moncur, J., \& Yonamine, D. (1977). Determinants of visitor expenditures and visitor lengths of stay: a cross-section analysis of U.S. visitors to Hawaii. Journal of Travel Research, 15(3), 5-8.

Mak, J., \& Nishimura, E. (1979). The economics of a hotel room tax. Journal of Travel Research, 17(4), 2-6. Martínez-Garcia, E., \& Raya, J. M. (2008). Length of stay for low-cost tourism. Tourism Management, 29(6), 1064-1075.

Menezes, A. G., Moniz, A., \& Vieira, J. C. (2008). The determinants of length of stay of tourists in the Azores. Tourism Economics, 14(1), 205-222.

Oppermann, M. (1994). Length of stay and spatial distribution. Annals of Tourism Research, 21(4), 834836.

Oppermann, M. (1995). Travel Life Cycle. Annals of Tourism Research, 22(3), 535-552.

Oppermann, M. (1997). First-time and repeat visitors to New Zealand. Tourism Management, 18(3), $177-$ 181.

Paul, B. K., \& Rimmawi, H. S. (1992). Tourism in Saudi Arabia: Asir National Park. Annals of Tourism Research, 19(3), 501-515.

Peypoch, N., Randriamboarison, R., Rasoamananjara, F., \& Solonandrasana, B. (2012). The length of stay of tourists in Madagascar. Tourism Management, 33(5), 1230-1235.

Raya, J. M., \& Martínez-Garcia, E. (2011). Nationality and low-cost trip duratio: a microeconometric analysis. Journal of Air Transport Management, 17(3), 168-174. 
Schoenfeld, D. A. (1982). Partial residuals for the proportional hazards regression. Biometrika, 69(1), 239241.

Seaton, A. V., \& Palmer, C. (1997). Understanding VFR tourism behaviour: the first five years of the United Kingdom Tourism Survey. Tourism Management, 18(6), 345-355.

Silberman, J. (1985). A demand function for length of stay: the evidence for Virginia Beach. Journal of Travel Research, 23(4), 16-23.

Sung, H. H., Morrison, A. M., Hong, G.-S., \& O'Leary, J. T. (2001). The effects of household and trip characteristics on trip types: a consumer behavioral approach for segmenting the U.S. domestic leisure travel market. Journal of Hospitality \& Tourism Research, 25(1), 46-68.

Tierney, P. T. (1993). The influence of state traveler information centers on tourist length of stay and expenditures. Journal of Travel Research, 31(3), 28-32.

UNWTO. (2006). Tourism Market Trends: World Overview \& Tourism Topics. Madrid: World Tourism Organization.

UNWTO. (2007). Tourism 2020 vision: global forecast. Madrid: UNWTO.

Uysal, M., McDonald, C. D., \& O'Leary, J. T. (1988). Length of Stay: A Macro Analysis For Cross-Country Skiing Trips. Journal of Travel Research, 26(3), 29-31.

Vaupel, J. W., Manton, K. G., \& Stallard, E. (1979). The impact of heterogeneity in individual frailty on the dynamics of mortality. Demography, 16(3), 439-454.

Walsh, R. G., \& Davitt, G. J. (1983). A demand function for length of stay on ski trips to Aspen. Journal of Travel Research, 21(4), 23-29.

Wurst, C. (1955). The length-of-stay problem in tourist studies. The Journal of Marketing, 19(4), 357-359.

Yang, Y., Wong, K. K. F., \& Zhang, J. (2011). Determinants of length of stay for domestic tourists: case study of Yixing. Asia Pacific Journal of Tourism Research, 16(6), 619-633.

Artigo recebido em: 07/05/2012. Artigo aprovado em: 27/08/2012. 